\title{
Generalized Theory of Monoligand-Receptor Binding for the Improvement of Nanoparticle Design
}

Ebtisam A. Aldaais*

Department of Biomedical Engineering, Imam Abdulrahman Bin Faisal University, Dammam, Saudi Arabia

\begin{abstract}
Superior design of therapeutic particles requires an understanding of the competition between electrostatic, van der Waals, and steric interactions in the system. This study combines those three forms of interactions and utilizes a decoupled mean-field approximation to generalize a molecular theory that determines the monoligand-receptor binding protocol. This protocol is designed to target receptors on cancer cells primarily through pH sensitivity and electrostatic binding while protecting healthy cells. The general theory of monoligand-receptor binding considers the influence of receptor densities on the binding and analyzes the molecular reorganization of two different kinds of grafted polymers (thermo-responsive and polybase) to a nanoparticle's surface that is near a cell surface. To enhance targeting, several stimuli and design parameters are studied to determine their effect on ligand complex elongation, which is crucial for binding efficiency. The results provide a design protocol (software) for effective ligand complexes for tumor targeting. In addition, the results indicate that controlling the salt concentration at the administrative site should advance the binding at the cancerous region.
\end{abstract}

Keywords: Monoligand-receptor; Polymer physics; Nanomedicine; Nanoparticle design; Meanfield theory; Intermolecular interactions

\section{Introduction}

The advantages of nanomedicine technology depend, to a large extent, on the creation of smart nanoparticles, such as micelles and liposomes. Micelles and liposomes are the best performing carriers as they can accommodate the delivery of diverse hydrophobic and hydrophilic drugs [1]. Smart nanoparticles can respond to a small range of external stimuli, such as small changes in temperature, $p H$, salt concentration, or magnetic and electrostatic field [2-5]. The nanoparticle's response appears as a change in the conformational structure that can be used to attach the nanoparticle to the targeted cell or release its contents at the site of administration. Smart nanoparticles are developed for drug delivery applications, biosensing and as molecular imaging tenders [6]. Moreover, nanoparticles are created from different kinds of polymers to protect their contents from the external environment, and achieve a desirable design to increase their capability of binding to the targeted cells [6-8].

The hydrophobicity of the nanoparticle's core, and the high toxicity of the hydrophobic anticancer drugs inspire biomedical engineers to design nanoparticles as anticancer drug delivery systems. An ideal nanoparticle's design for cancer treatment should prevent any drug leakage, and ensure the delivery to cancerous cells only. In 2006, Nasongkla et al. developed a multifunctional micelle to target integrin $\alpha_{v} \beta_{3}$ in cancer cells, and deliver doxorubicin (DOX) and MRI-ultrasensitive particles to the tumor [6]. Dhar et al. found a unique micellar design that is made of poly(D,L-lactic-co-glycolic acid) (PLGA)-poly(ethylene glycol) (PEG) to deliver an anticancer drug (cisplatin) to prostate cancer cells [7]. The polymers are attached to prostate-specific membrane antigen (PSMA) targeting aptamers. Their specific design ensured the binding to prostate cells, and the release of the drug inside the cells through endocytosis. Another study by Ahn et al. used antibody-drug congregates (ADCs) for targeted delivery, such that ADCs ligands are attached to micelles that are loaded with cytotoxic platinum drugs for pancreatic tumor treatment [8]. Their results show efficient delivery of the anticancer drug, and accordingly a significant suppression in the growth of the pancreatic malignant cells.
Despite the development of the nanoparticle's designs for targeted delivery to cancer cell, a review paper by Wilhelm et al. stated that, "after surveying the literature from the past 10 years, only $0.7 \%$ (median) of the administered nanoparticle dose is found to be delivered to a solid tumor" [9]. Therefore, refining nanoparticles that can target malignant cells with very low concentration compared to healthy cells in the blood stream is sufficient motivation for the further development of smart nanoparticles. Improving the targeting proficiency of smart nanoparticles is governed by the choice of polymers that are attracted to malignant cells only, and ligands that bind to cancer cells through the molecular recognition of specific cancer markers [10]. Several theoretical studies attempted to understand the ligand-receptor binding properties through consideration of the molecular interaction in the biological system $[11,12]$. One revealed that attaching desired ligands to polybases in a nanoparticle has improved the ligand-overexpressed receptor binding in cancer cells [13]. Evidently, the positively charged segments on the polybases improve the targeting as several cancer cells have negatively charged lipids [14,15].

The monoligand-recptor binding theory presented here models the molecular reorganization of grafted thermo-responsive polymers and polybases to a surface of a nanoparticle that is near a cell surface (dense system) on a cubic lattice (Figure 1). The thermo-responsive polymers create the shell of the nanoparticle such that their length, density, and type are significant design parameters of the nanoparticle [16]. The charged polybases are electrostatically attracted to the negatively charge surface of the cancer cell $[14,15,17]$. The developed molecular theory, a general theory of monoligand-receptor binding behavior,

${ }^{*}$ Corresponding author: Ebtisam A. Aldaais, Department of Biomedical Engineering Imam Abdulrahman Bin Faisal University, Dammam, P.O. Box-243531451, Saudi Arabia, Tel: 966133331703; E-mail: ealdaais@iau.edu.sa

Received: July 13, 2018; Accepted: July 28, 2018; Published: July 31, 2018

Citation: Aldaais EA (2018) Generalized Theory of Monoligand-Receptor Binding for the Improvement of Nanoparticle Design. J Nanomed Nanotechnol 9: 512. doi: 10.4172/2157-7439.1000512

Copyright: (c) 2018 Aldaais EA. This is an open-access article distributed under the terms of the Creative Commons Attribution License, which permits unrestricted use, distribution, and reproduction in any medium, provided the original author and source are credited. 


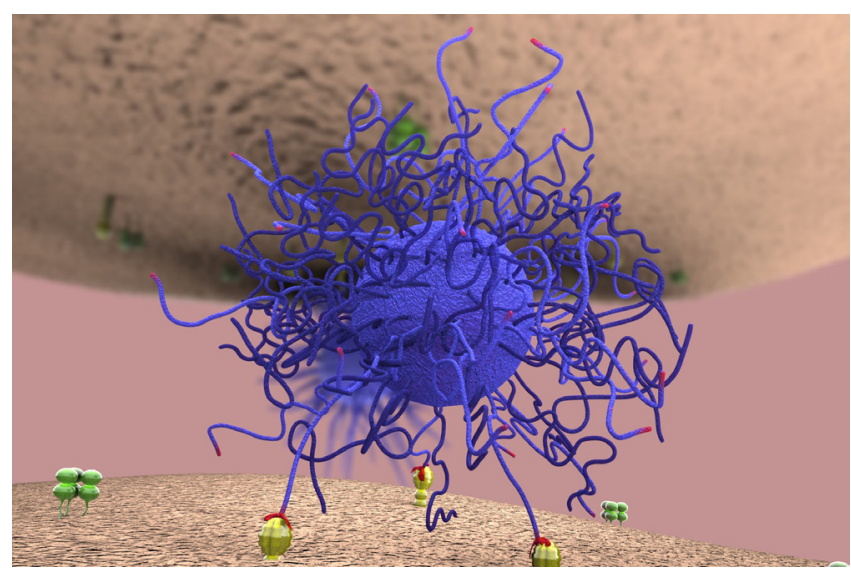

Figure 1: An illustration of a nanoparticle that has shell-forming polymers (dark blue lines) and polybases (light blue lines) that are attached to ligands (red tips). It illustrates the monoligand-receptor binding between the ligands and specific receptors (yellow) on the cell surface. Note that the cell surface contains other receptors (green).

predicts the competition between van der Waals, electrostatic, and steric interactions in a very dense system using a decoupled mean-field approximation. The competition between these forces leads to two significant therapeutic states. The shield state that represents collapsed polybases to protect the ligands from binding to healthy cells when the nanoparticle is distant from the cancer cell, while the exposed state represents the stretched polybases that expose the ligands to bind to specific receptors on the malignant cells. The focus of this study is to improve the second state, which depends on the shell-forming polymer "thermo-responsive polymers" and ligand complex "polybase attached to ligands" elongation under several biological conditions and nanoparticle's design.

The monoligand-receptor binding system is a very dense system that is composed of at least nine different molecules; water molecules (w) $, \mathrm{H}^{+}, \mathrm{OH}^{-}$salt ions $\left(\mathrm{Na}^{+}, \mathrm{Cl}^{-}\right)$, monomers of the shell-forming polymers $(S)$, uncharged and charged monomers that form the ligand complexes $\left(L, L_{+}\right)$, and bound ligand-receptors $(L R)$. These molecules interact with each other via steric, van der Waals, and electrostatic interactions. This work aims to improve the monoligandreceptor binding by understanding the molecular interactions and reorganization between these molecules under several environmental conditions. The general molecular theory uses a decoupled mean-field approximation approach, which was developed in [18], not only to easily explore a large parameter space that cannot be done via simulation, but also to improve the accuracy in analyzing the molecular selfreorganization of grafted shell-forming polymers and ligand complexes. The system is modeled on a cubic lattice with coordination number six. Details about the system's design and properties are discussed in the Model section. The generalized theory is developed through the determination of the free energy in the system, which is discussed in the Theoretical Approach section. The Result section examined the effect of several stimuli (such as local temperature, $p H$, and cell surface charge) and design parameters (such as polymers density and fraction of ligands) on stretching the ligand complexes. Finally, a brief conclusion about the monoligand-receptor binding properties is reviewed with suggestions for future work to enhance the fraction of ligand-receptor binding.

\section{Model}

The model is designed to focus on the molecular reorganization between a nanoparticle surface and a cell surface, where both are considered as planar. The system between the two planar contains water molecules $(w), \mathrm{H}^{+}, \mathrm{OH}^{-}$salt ions $\left(\mathrm{Na}^{+}, \mathrm{Cl}^{-}\right)$, and several segments of two different kinds of polymers. Figure 2 represents two kinds of grafted polymers to a nanoparticle's surface that interact with a cell surface. The two different kinds of grafted polymers have a total density, and number of monomers for both.

The first kind of polymers is shell-forming polymers (illustrated by dark blue lines). As these polymers form the nanoparticle shell, their length can affect the overall nanoparticle size, as well as their thermoresponse. Shell-forming polymers that collapse at body temperature reduce the nanoparticles size and vice versa. Also, distributing these uncharged polymers on the nanoparticle surface reduces the unfavorable electrostatic interaction between different charged ligand complexes, while strengthening the repulsion between different charged segments from the same ligand complex, which should lead to the desired stretching of the ligand complex. Hydrophilic-biocompatible shell-forming polymers can be used as a non-inflammatory modifier for drugs. The choice of shell-forming polymers should serve to increase the solubility and improve the biocompatibility of the therapeutic nanoparticle.

The second kind of polymers that are attached to the nanoparticle's surface is polybases (illustrated by light blue lines with white rings (charges)). These polymers are attached to ligands illustrated by the red tips. By definition, polybases are polyelectrolytes that have basic groups, which are able to bind to free hydrogen ions $\left(\mathrm{H}^{+}\right)$in the biological aqueous environment and become positively charged with an association constant $K_{a}$ (note that $L$ represents uncharged segments and $L_{+}$represents charged segments). The positive segments on the polybases $\left(L_{+}\right)$become attracted to negatively charged surface of the

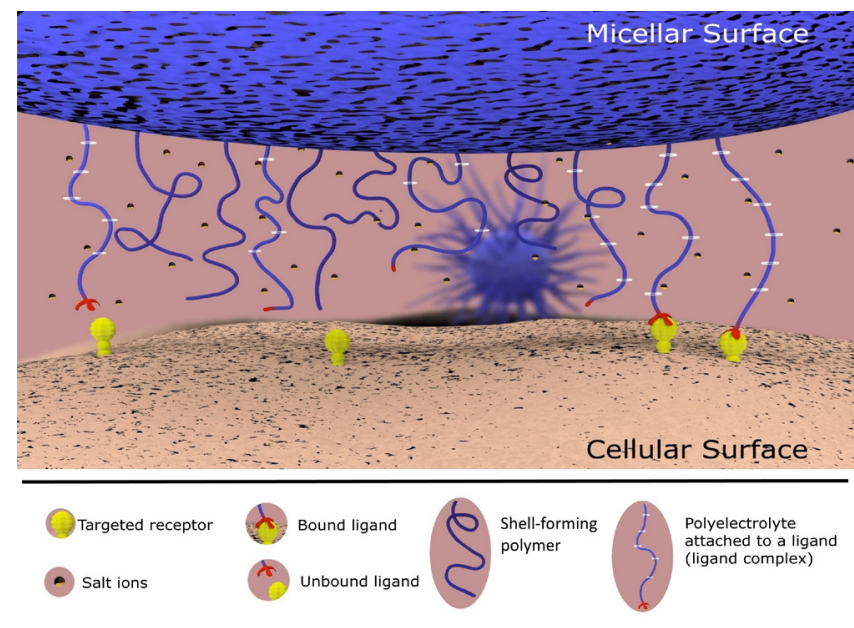

Figure 2: An illustration of the system under study, which is submerged in an aqueous solution and bounded by the surfaces of the nanoparticle and the cell. Both surfaces are taken to be planar. The nanoparticles's surface holds two different kinds of polymers: shell-forming polymers and ligand complexes (polybases that are charged (white rings) and attached to ligands (red tips)), while the cell surface holds a specific density of the targeted receptors. The system is studied under several biological conditions to determine the design parameters that enhance the monoligand-receptor binding behavior. 
cancerous cells, allowing ligand complexes to stretch and expose their ligands to the receptors $(R)$ on the cell surface, and hence the binding probability increases $[14,15]$. The ligand-receptor $(L R)$ binding can be described by the reaction $L+R \rightleftharpoons L R$, and a binding association constant

$$
K_{L R}=\frac{[L R]}{[L][R]}=C \exp \left(-\beta\left(\mu_{L R}^{\circ}-\mu_{L}^{\circ}-\mu_{R}^{\circ}\right)\right) .
$$

The density of targeted receptors on the cell surface is represented by. The fraction of ligand complexes on the nanoparticle surface is given by $X_{L}=\frac{N_{L}}{N_{L}+N_{S}}$, where $N_{L}$ and $N_{S}$ refers to number of ligand complexes and shell-forming polymers respectively. The fraction of bound ligand complexes is $f_{L R}=\frac{[L R]}{[L R]+[L]}$, where $[L R]$ and [L] are the concentration of bound and free ligand complexes respectively [12]. Correspondingly, a unit area of the system has a concentration of:

- shell-forming polymer segments $\left(\sigma_{S}\right)=\sigma\left(1-X_{L}\right)$

- free ligand complex segments $\left(\sigma_{L}\right)=\sigma X_{L}\left(1-f_{L R}\right)$

- bound ligand complex segments $\left(\sigma_{L R}\right)=\sigma X_{L} f_{L R}$

- free receptors $=\sigma_{R}-\sigma X_{L} f_{L R}$

The space between the two planar surfaces of the nanoparticle and target cell is modeled as a cubic lattice, where each molecule occupies a single cubic lattice site. Each polymer's configuration $(\alpha)$ is built randomly using self avoiding random walk (SAW) with Rosenbluth \& Rosenbluth system of weights to improve the statistics [19]. The system is treated as incompressible. The compressibility constraint maintains the repulsive steric interactions between all molecules in the system; water molecules $(w), \mathrm{H}^{+}, \mathrm{OH}^{-}$salt ions $\left(\mathrm{Na}^{+}, \mathrm{Cl}^{-}\right)$, shell-forming polymer segments $(S)$, uncharged and charged ligand complex segments $\left(L, L_{+}\right)$, and bound ligand-receptors $(L R)$.

The average volume fraction of polymer $p=\{S, L, L R\}$ segments at layer $z\left(\left\langle\phi_{p}(z)\right\rangle\right)$ is defined as:

$$
\left\langle\phi_{p}(z)\right\rangle=\sigma \sum_{\alpha} P_{p}(\alpha) n_{p}(\alpha, z) v
$$

where, $P_{p}(\alpha)$ is the probability of having polymer $p$ in a configuration $\alpha, n_{p}(\alpha, z)$ is the number of polymer segments at layer $z$, and $v$ is the volume of each segment, which is chosen to be equal to the volume of the solvent molecule for simplicity. Notice that $n_{p}(\alpha, z) v=v_{p}(\alpha, z)$ is another notation that will be used in the Theoretical Approach section and represents the volume fraction of polymer $p$ segments. All other molecules have a volume fraction $\phi_{j}(z)$, where $j=\left\{w, H^{+}, \mathrm{OH}^{-}, \mathrm{Na}^{+}(\right.$or +$), \mathrm{Cl}^{-}($or -$\left.)\right\}$. The associated functional groups on the ligand complexes (polybases) gain an amount of charge, while the other charged species in the system have $q_{j}$ charge. Thus, the average density of charged molecules at layer $z\left(\left\langle\rho_{q}(z)\right\rangle\right)$ is equal to the sum of the average density of charged segments and the sum of densities of all other charged species:

$$
\left\langle\rho_{q}(z)\right\rangle=f_{H^{+}}(z) q_{p}\left(\left\langle\rho_{L}(z)\right\rangle+\left\langle\rho_{L R}(z)\right\rangle\right)+\sum_{j} q_{j} \rho_{j}(z)
$$

Here, $f_{H^{+}}$is the protonation fraction.
Each molecule in the system is subject to van der Waals and electrostatic forces. The van der Waals interactions are calculated for each configuration $(\alpha)$ through the intra-molecular interactions $\left(E_{\text {intra }}(\alpha)\right)$ between segments of the same polymer, and the intermolecular interactions $\left(E_{\text {inter }}(\alpha)\right)$ between polymer segments from different polymers. The values of their two elementary short-ranged molecular-attractive interactions, $\left(\varepsilon_{\text {intra }}\right.$ and $\left.\varepsilon_{\text {inter }}\right)$, are chosen so as not to affect the solvent solubility. A decoupled mean-field approach is used to improve the accuracy in calculating the inter-molecular interactions $[18,20,21]$.

The electrostatic interactions between two charged molecules, such as salt ions and charged polymer segments, are influenced by several environmental and design parameters. These parameters include the cell surface charge density, salt concentration, local $p H$, polymers density, and polybase association constant. Several biological conditions and design parameters are examined in this model to predict distinctive electrostatic interactions. Different electrostatic interactions, that are represented by electric potential profile $(\psi(z))$, cause different configurations and accordingly changes the intra and inter-molecular interactions to stabilize the system.

This system undergoes several chemical interactions (formation of charged ions $\left(\mathrm{H}^{+}, \mathrm{OH}^{-}, \mathrm{Na}^{+}, \mathrm{Cl}^{-}\right)$, charged polymer segments $\left(L_{+}\right)$, and ligand-receptor binding $\left.(L R)\right)$. The model incorporates the complexity of the chemical equilibrium and the physical interactions (van der Waals, electrostatic, and steric interactions) between all molecules in the system. While considering the chemical equilibrium and physical interactions in the system, this model allows one to examine the effect of several biological and design parameter on the elongation of the ligand complexes and accordingly the ligandreceptor binding probability.

\section{Theoretical Approach}

The space between the nanoparticle and the cell surfaces is discretized into layers in the $X Y$ plane with thickness $d z$. In this model, it is assumed that the system is homogeneous in planes parallel to the cell surface or the nanoparticle surface $(x y)$, and inhomogeneous in the norm direction $(z)$ [22]. The average volume fraction of shellforming polymers, ligand complexes, and ligand complexes that are bound to receptors are represented by $\left\langle\phi_{S}(z)\right\rangle,\left\langle\phi_{L}(z)\right\rangle$, and $\left\langle\phi_{L R}(z)\right\rangle$ respectively, while $\phi_{j}(z)$ is the volume fraction of specie $j=\left\{w, \mathrm{H}^{+}, \mathrm{OH}^{-},+\left(\mathrm{Na}^{+}\right),-\left(\mathrm{Cl}^{-}\right)\right\}$. The system is subject to the compressibility constraint:

$$
\begin{aligned}
& \left\langle\phi_{S}(z)\right\rangle+\left\langle\phi_{L}(z)\right\rangle+\left\langle\phi_{L R}(z)\right\rangle+\phi_{w}(z)+ \\
& \phi_{H^{+}}(z)+\phi_{O H^{-}}(z)+\phi_{+}(z)+\phi_{-}(z)+\phi_{R}(z)=1
\end{aligned}
$$

The system's Helmholtz free energy per unit area is a sum of several terms; conformational entropy $\left(F_{\text {conf }}\right)$, van der Waals interactions $\left(F_{v d w}\right)$, electrostatic interactions $\left(F_{\text {elc }}\right)$, ligand-receptor binding energy $\left(F_{L R}\right)$, ion formation energy $\left(F_{i f}\right)$, mixing (translational) entropy $\left(F_{m i x}\right)$, and chemical interactions $\left(F_{c h e m}\right)$.

$$
\frac{F}{A}=\frac{F_{c o n f}+F_{v d w}+F_{e l c}+F_{L R}+F_{i f}+F_{m i x}+F_{c h e m}}{A}
$$


Within self-consistent field theory, the most probable configurations of shell-forming polymers $\left(P_{S}(\alpha)\right)$, free ligand complexes and bound ligand complexes $\left(P_{i}(\alpha)\right)$, where $i \equiv\{L, L R\}$ were determined. By minimizing the free energy and introducing Lagrange multipliers $\pi(z)$ to implement the local incompressibility constraint, one obtains the following probabilities which completely describe the system:

$$
\begin{aligned}
P_{S}(\alpha)= & \frac{W_{R}}{\mathbb{Z}_{S}} \exp \left(-\beta\left[E_{\text {intra }}(\alpha)+E_{\text {inter }}(\alpha)\right]-\beta \int \pi(z) v_{S}(\alpha, z) d z\right)(5) \\
P_{i}(\alpha)= & \frac{W_{R}}{\mathbb{Z}_{i}} \exp \left(-\beta\left[E_{\text {intra }}(\alpha)+E_{\text {inter }}(\alpha)\right]-\beta \int \pi(z) v_{i}(\alpha, z) d z-\right. \\
& \left.\beta \int n_{i}(\alpha, z)\left[q_{p} \psi(z)-\ln f_{H^{+}}(z)\right] d z\right)
\end{aligned}
$$

Here, $\mathbb{Z}$ is the partition function of the corresponding polymer, and it satisfies the condition that $\sum_{\alpha} P(\alpha)=1$. The RosenbluthRosenbluth weight $W_{R}$ is used to improve the statistics of the polymer's possible configurational structures.

$$
W_{R}=\prod_{m=1}^{N}\left(\frac{D_{m}}{5}\right)
$$

The system of weights is calculated while building the polymer chain that has $N$ monomers $(m=1: N)$. The variable $D_{m}$ is the number of available positions for the next monomer, from which the actual position is chosen randomly. $D_{m}$ is divided by the maximum number of available positions for SAW on a cubic lattice with a coordination number of six [19].

The first two terms in all probabilities account for van der Waals interactions. They incorporate the intra and inter-molecular interactions between polymer segments from the same polymer chain, and polymer segments and their neighbor molecules respectively. $\beta=\frac{1}{K_{B} T}$ is the inverse thermodynamic temperature. For simplicity, it's assumed that polymer segments from different polymer chains interact with each other as they interact with the solvent molecules. For each configuration $(\alpha)$, one can calculate the polymer intramolecular interaction energy $\left(E_{\text {intra }}(\alpha)\right)$ by calculating the number of neighboring unbound monomers within a polymer chain. This can be done by calculating the distance between monomer $i$ and

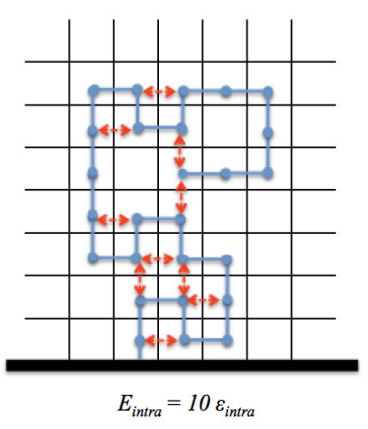

$$
\begin{array}{l|l|l|l|l|l|l} 
& 1 & 1 & 1 & 1 & 1 & \\
\hline 1 & & & & & & 1 \\
\hline 1 & & & & 4 & & 1 \\
\hline 1 & & 4 & & & & 1 \\
\hline 1 & & & & 3 & 1 & \\
\hline 1 & & & & & 1 & \\
\hline & 2 & & & & 1 & \\
\hline & 1 & & & & 1 & \\
\hline \eta(i, j) \text { at each point around a configured } \\
\text { polymer. }
\end{array}
$$

Figure 3: A 2D illustration of a random SAW that is used to demonstrate a polymer chain. The left side illustration shows how to determine the exact number of intra-molecular interactions, while the right side illustrates the possible inter-molecular interactions at several points around the same chain. monomer $i+3$ in the same chain, and placing a constraint on that distance. When the constraint equals one, intra interaction energy is added to the chain at that specific configuration (Figure 3 ). Thus, we define $E_{\text {intra }}(\alpha)$ for a specific configuration $\alpha$ mathematically by the following relation:

$$
E_{\text {intra }}(\alpha)=-\varepsilon_{\text {intra }} \sum_{n=1}^{N} \sum_{N}^{N} \delta_{1, r}
$$

where $r$ is the distance between monomers $m$ and $n, \varepsilon_{\text {intra }}$ represents the elementary intra attractive energy, and $\delta_{1, r}$ is a Kronecker delta that equals unity when $r=1$ and otherwise.

A decoupled self-consistent theory is applied to calculate the intermolecular interactions energy $\left(E_{\text {inter }}\right)[18,20,21]$. To increase the efficiency of this calculation, all possible inter-molecular interactions for each monomer were traced while building the chain by using SAW. Each new monomer added to the chain should have a number of possible positions, one of which is chosen for a specific configuration, while the others are stored as possible inter-molecular interaction sites at the $z$ layer $\eta(\alpha, z)$. Following the decoupled mean-field approach that was proposed in [18], the inter-molecular interaction energy depends not only on the average volume fraction of polymer segments at layer $z(\langle\phi(z)\rangle)$, but also the average number of inter-molecular interactions $(\langle\eta(z)\rangle)$ :

$$
\left\langle E_{\text {inter }}\right\rangle=-\frac{\varepsilon_{\text {inter }}}{2}\langle\phi(z)\rangle\langle\eta(z)\rangle d z
$$

Figure 3 shows the possible inter-molecular interaction for specific configuration in two-dimensions.

In the third term of both equations (5) and (6), $v_{S}(\alpha, z)$ and $v_{i}(\alpha, z)$ are the volume fractions of shell-forming polymer segments, and free or bound ligand complex segments that occupy layer $z$.

The last two terms in equation (6), account for the electrostatic interactions as this equation describes the probability of the free and bound ligand complexes that are made of polybases. In the fourth term of equation (6), $q_{p}$ is the amount of charge on an ionized monomer, and $n_{i}(\alpha, z)$ is a function that counts all segments of polymer $i$ at layer $z$. In the same term, $\psi(z)$ is the electric potential at layer $z$. In the last term of equation (6), $f_{H^{+}}$is the fraction of charged monomers, which is obtained by the following:

$$
\frac{f_{H^{+}}(z)}{\left(1-f_{H^{+}}(z)\right)}=\frac{\phi_{H^{+}}(z)}{K_{a}^{\circ} \phi_{w}(z)}
$$

The volume fraction profile of all molecular species in the system is given by minimizing the free energy with respect to the corresponding $\phi_{j}(z)$ :

$$
\begin{aligned}
& \phi_{w}(z)=\exp \left(-\beta \pi(z) v_{w}\right) \\
& \phi_{H^{+}}(z)=\exp \left(-\beta \mu_{H^{+}}^{\circ}-\beta \pi(z) v_{w}-\beta q_{H^{+}} \psi(z)\right) \\
& \phi_{O H^{-}}(z)=\exp \left(-\beta \mu_{O H^{-}}^{\circ}-\beta \pi(z) v_{w}-\beta q_{O H^{-}} \psi(z)\right) \\
& \phi_{+}(z)=\exp \left(-\beta \mu_{+}-\beta \pi(z) v_{w}-\beta q_{+} \psi(z)\right) \\
& \phi_{-}(z)=\exp \left(-\beta \mu_{-}^{\circ}-\beta \pi(z) v_{w}-\beta q_{-} \psi(z)\right)
\end{aligned}
$$


where, $\mu_{j}^{\circ}$, and $q_{j}$ are the standard chemical potential, and the amount of charge for specie $j$ respectively. All molecules in the system are assumed to have the same volume as the solvent molecule (water).

The fraction of ligand-receptor binding is obtained from the system's free energy:

$$
\frac{f_{L R}}{\left(1-f_{L R}\right)}=\frac{C K_{L R} \mathbb{Z}_{L R} e}{\mathbb{Z}_{L} \phi_{R} n_{R}}\left(1-\frac{\sigma X_{L} f_{L R}}{\sigma_{R}}\right)
$$

where, $\mathbb{Z}_{L}$ and $\mathbb{Z}_{L R}$ are the partition functions for free and bound ligand complexes. Also, is the constant in the association constant equation $K_{L R}=C \exp \left(-\beta\left(\mu_{L R}^{\circ}-\mu_{L}^{\circ}-\mu_{R}^{\circ}\right)\right), n_{R}$ is the number of receptors on the interacting cell surface at a specific microstate, and $e$ is the base of natural logarithm.

The system is discretized into $z$ layers with thickness of $0.3 \mathrm{~nm}$. The non-linear system is composed of seven sets of unknowns. Three of these unknowns are the average volume fractions of shell-formers $(S)$, free ligand complexes $(L)$, and bound ligand complexes $(L R)\left(\left\langle\phi_{S}(z)\right\rangle,\left\langle\phi_{L}(z)\right\rangle,\left\langle\phi_{L R}(z)\right\rangle\right)$. Note that both free and bound ligand complexes have segments at each layer, however only the bound ones have segments on the cell surface. Another three unknowns are the average fraction of inter-molecular interactions for $S, L$, and $L R$ segments $\left(\left\langle\eta_{S}(z)\right\rangle,\left\langle\eta_{L}(z)\right\rangle,\left\langle\eta_{L R}(z)\right\rangle\right)$. The final unknown is the electric potential profile $(\psi(z))$. The incompressibility constraint and the definitions of $\left(\left\langle\phi_{L}(z)\right\rangle,\left\langle\phi_{L R}(z)\right\rangle,\left\langle\eta_{S}(z)\right\rangle,\left\langle\eta_{L}(z)\right\rangle,\left\langle\eta_{L R}(z)\right\rangle\right)$ are used with Poisson equation to solve for the seven sets of unknowns. Exterminimizing the free energy with respect to the electric potential one obtains Poisson equation in the following form:

$$
\frac{d^{2} \psi(z)}{d z^{2}}=-\frac{\left\langle\rho_{q}(z)\right\rangle}{\epsilon}
$$

where $\epsilon$ is the permittivity of the medium, which we assume to be water. $\left\langle\rho_{q}(z)\right\rangle$ is the density of charges in the system, which is defined by Equation [2]. The electric potential profile is subject to two boundary conditions: the charge density on the cell surface $\sigma_{q_{\text {cell }}}$ and the charges on the nanoparticle's surface $\sigma_{q_{N P}}$, which depends on the number of charged polymer segments that are attached to that surface.

$$
\frac{\partial \psi(z)}{\partial z}=\left\{\begin{array}{lll}
\frac{-\sigma_{q_{N P}}}{\epsilon}, & \text { for } & z=1 \\
\frac{-\sigma_{q_{\text {cell }}}}{\epsilon}, & \text { for } & z=L
\end{array}\right.
$$

The Poisson equation is solved by determining the total charge density on both the nanoparticle's surface and the cell surface. The fraction of charged monomers on both surfaces is considered in calculating the total charge density. We calculate the fraction of charged monomers when the cell surface is far away, $\left.\frac{\partial \psi(z)}{\partial z}\right|_{z=L}=0$, and use the results as an initial guess to solve the system with a close cell surface. In addition to the constant charge density, the total charge densities on the surfaces, $\left(\sigma_{q_{N P}}, \sigma_{q_{\text {cell }}}\right)$, are affected by the density of ligand complexes, dissociation constant, local $p H$, and salt concentration.

\section{Results and Discussion}

The system is modeled on a cubic lattice and discretized into layers on the $X Y$ plane with $0.3 n m$ thickness $\left(0.3 n m \simeq \sqrt[3]{v_{w}}\right)$. Both kinds of polymer chains in the system, which are thermo-responsive (shell-forming) polymers and polybases (ligand complexes), consist of 25 monomers. The electric potential profile at two different salt concentrations that have two different Debye lengths is determined to ensure the validity of the theory. Figure 4 shows $\psi(z)$ at $C_{\text {salt }}=0.1 \mathrm{M}$ and $0.4 \mathrm{M}$ with $\lambda_{D} \simeq 1 \mathrm{~nm}$ and $\simeq 0.5 \mathrm{~nm}$ respectively at three different conditions. The first condition represents no charge on the cell surface. The nanoparticle's surface has positively charged segments from the grafted polyelectrolytes. Thus, the electric potential profile decreases dramatically from a positive electric potential value at the nanoparticle surface $\left(\psi_{\max } \sim 10 \mathrm{mV}\right.$ at $C_{\text {salt }}=0.1 \mathrm{M}$ and $\psi_{\max } \sim 4 \mathrm{mV}$ at $\left.C_{\text {salt }}=0.4 \mathrm{M}\right)$

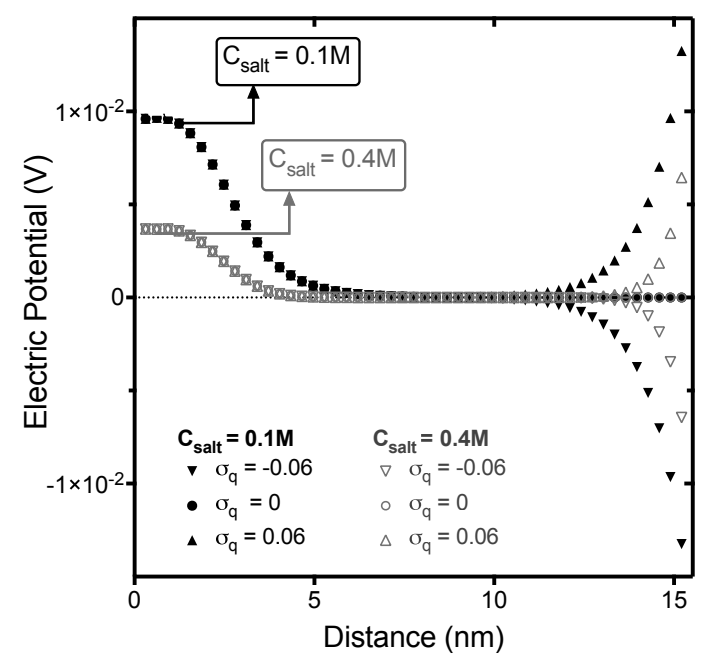

Figure 4: The electric potential profile for the same density of grafted polybases $\left(\sigma=3 / \mathrm{nm}^{2}, X_{L}=0.4\right)$ and different charges on the surface of the targeted cell at $C=0.1 \mathrm{M}$ (blck plots and $\lambda_{\mathrm{D}} \sim 1 \mathrm{~nm}$ ) and $0.4 \mathrm{M}$ (gray plots and $\lambda_{\mathrm{D}} \sim 0.5 \mathrm{~nm}$ ) Increasing the salt concentration decreases the Debye length and accordingly the screening effect.
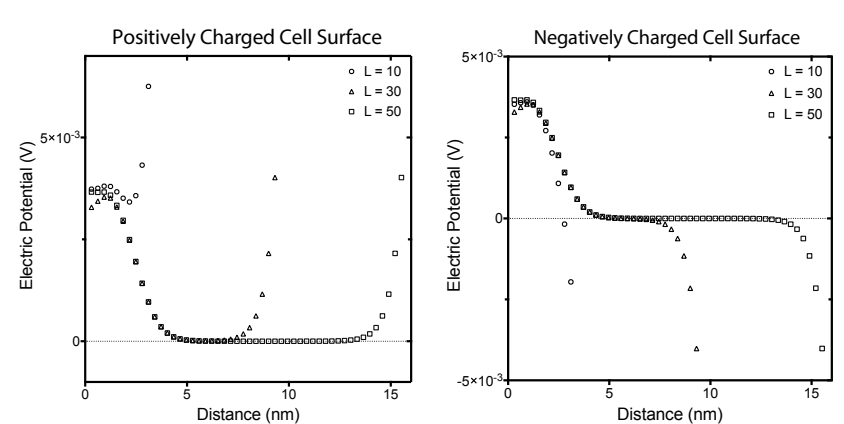

Figure 5: The zero domain at the electric potential profile for both negatively and positively charged cell surfaces is affected by the distance between the nanoparticle and the cell. When the fraction of $\lambda_{D}$ and this distance is less than 0.125 , both cases have no zero domain and accordingly no bulk behavior. 
to reach absolute zero arround 10 and $6.6 \mathrm{~nm}$ respectively and continue to be at the cell surface.

The second and the third conditions illustrate a positive and negative charge density on the cell surface at $\sigma_{q}=0.06$ and $-0.06 \mathrm{e} / \mathrm{nm}^{2}$ respectively. In both conditions and for the two different salt concentrations, the electric potential profile shows at first an asymptotic convergence from highly ionized region to more diffuse region with less charges until it reaches zero at an intermediate distance where there are no polyelectrolyte segments, and no effect of the charge on the cell surface. This zero domain is affected by the salt concentration, the charge density, and the distance between the nanoparticle's surface and the cell surface (Figure 5). The electric potential passes the zero domain to increase or decrease as it approaches the positively or negatively charged cell surface respectively.

The black plots in Figure 4 show that reducing the salt concentration to $C_{\text {salt }}=0.1 \mathrm{M}$ (with Debye length $\lambda_{D}=0.962 \mathrm{~nm}$ ), causes higher accumulation of the electric ions near both the nanoparticle's surface and the cell surface in comparison to the case of higher salt concentration at $C_{\text {salt }}=0.4 M$ (with Debye length $\lambda_{D}=0.490 \mathrm{~nm}$ ) (gray plots). Due to the high ionic strength at $C_{\text {salt }}=0.1 M$, the electrostatic interactions between polymer segments are mainly screened.

Figure 5 demonstrates that when the fraction of Debye length to the separation distance between the nanoparticle surface and the cell surface is below 0.125 , there is no zero domain; hence no bulk behavior. The system is studied at $3 \mathrm{~nm}$ distance (tenth layer), which is around the average length of the polymers, where the ligand-receptor binding appears. Therefore, the ligand-receptor binding behavior appears in a system with no bulk properties, and the fraction of charged monomers $\left(f_{H^{+}}(z)\right)$ vary greatly between the layers with small changes in the biological parameters, such as $p H$ and $C_{\text {salt }}$. Their values influence the charged monomers position and accordingly ligand complex elongation.

It's obvious that the ligand complex elongation and the polymer's segments accumulation influence the ligand-receptor binding. These two are affected by several environmental parameters, such as the local temperature, local $p H$, and cell surface charge. They are also affected by several engineered parameters, such as the density of polymers on
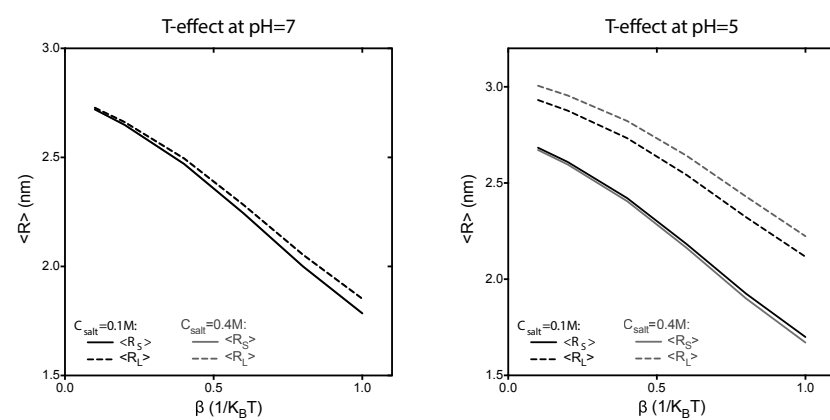

Figure 6: The effect of the temperature on the length of the shell-forming polymers and ligand complexes at two different $\mathrm{pH}$ and salt concentrations. Both polymers stretch at low $\beta$ (high $\mathrm{T}$ ). However, at neutral system with $\mathrm{pH}=7$, changing the salt concentration has no effect on the plots as the hydrogen and hydroxide ions are balanced. On the other side, at $\mathrm{pH}=5$, ligand complexes stretch more at $C_{\text {salt }}=0.4 \mathrm{M}$ (see gray dashed line). Changing the salt concentration shows a slight effect on the shell-forming polymers due to the steric forces, such that the solid gray line (at $\left.C_{\text {salt }}=0.4 M\right)$ falls under the solid black line $\left(\right.$ at $\left.C_{\text {salt }}=0.1 \mathrm{M}\right)$ the nanoparticle. To present the importance of the theory as a design guideline, the effect of each parameter is studied below.

\section{Local temperature}

The theory captures polymers that extend at temperatures above their collapse temperature (usually chosen around the body temperature) and collapse at temperatures below it. In Figure 6, both the shell-forming polymers (thermo-responsive) and ligand-complexes (polybases) collapse at low local temperature. We vary the system's temperature by changing $\beta$ values between 0.1 and 1.0, while keeping the elementary molecular attractive interaction $\left(\varepsilon_{\text {intra }}, \varepsilon_{\text {intra }}\right)$ at a value of $-1 K_{B} T$. Decreasing temperatures values shows a decrease on the polymer's average length $\langle R\rangle$, which is an expected entropic effect. Although both the shell-forming polymers and ligand complexes have the same number of segments, the ligand complexes show greater extension. Their plot (dashed line) is above the shell-forming polymers' plot (solid line). The positively charged polymer segments on the ligand complexes create repulsive interactions between them, which extend the polymer's length.

The left side graph of Figure 6 shows the temperature effect for two different salt concentrations when the system's local $p H$ is at 7 . The solid black and gray plots for $\left\langle R_{S}\right\rangle$ at $C_{\text {salt }}=0.1$ and $0.4 M$ respectively are overlapping. Also, the dashed black and gray plots for $\left\langle R_{L}\right\rangle$ at $C_{\text {salt }}=0.1$ and $0.4 \mathrm{M}$ respectively are overlapping as well. As a simple entropic effect, decreasing the temperature is shortening the chains; however changing the salt concentration doesn't have a considerable effect on polymer elongation at a local $p H$ of 7 (for polymers with $p K_{a}=5$ ). The right side graph of Figure 6 shows how changing the salt concentration affects the elongation of the ligand complexes at local $p H$ of 5 . Increasing the salt concentration $\left(C_{\text {salt }}=0.4 \mathrm{M}\right)$ should increase the screening effect and decrease the repulsive interactions between polymer's segments causing a decrease on its length. However this is not what we see in the dashed gray line

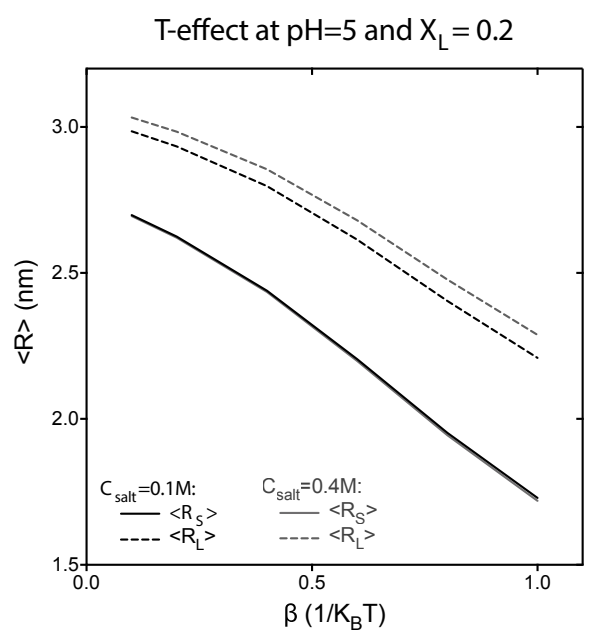

Figure 7: Decreasing the fraction of ligand complexes from 0.4 to 0.2 diminishes the effect of the salt concentration on the shell forming polymers that we have observed in the previous figure (Figure 6) - the two plots of the shell-forming polymers $\left\langle R_{S}\right\rangle$ at the two different salt concentrations (solid lines) are overlapping. 
at the right side graph of Figure 6. In this case, the polybases stretch at higher salt concentration as the salt ions accumulate at the charged planes

The solid gray plot on the right side of Figure 6 for $\left\langle R_{S}\right\rangle$ at $C_{\text {salt }}=0.4 \mathrm{M}$ falls slightly below the case of lower salt concentration (solid black line). Shell-forming polymers don't have charged segments. Thus, they are not affected by the change of salt concentration or $p H$ directly, but they are affected by van der Waals forces and the steric volume effect. Apparently, these two are changed in the system due to the change of ligand complex configurations. Accordingly, shell-forming polymer configurations are affected. The stretched ligand complexes shorten the shell-forming polymers. This effect decreases as the density of the ligand complexes decreases. Figure 7 shows the elongation of the two kinds of polymers in the system at a local $p H$ of 5 and 0.2 fraction of the ligand complexes, which was 0.4 in the previous figure. The elongation plots of the shell-forming polymers at the two different salt concentrations in Figure 7 are overlapping.

\section{Local}

As mentioned earlier, low salt concentration causes an increase in the Debye length. When the Debye length is about $1 \mathrm{~nm}$, there are no electrostatic interactions between polymer segments; they are mainly screened. Thus, the local $p H$ effect was studied at high $C_{\text {salt }}$, when Debye length is about $0.5 \mathrm{~nm}$. Also, the effect of local $p H$ on the polymer's length is subject to the polyelectrolyte's $p K_{a}$ value. The average end-to-end distance for ligand complexes $\left\langle R_{L}\right\rangle$ is calculated at different $p K_{a}$ values $\left(p K_{a}=5: 8\right)$ when the nanoparticle is far from the cell $(L=50)$. Accordingly, the effect of the charge on the cell surface is neglected $\left(q_{\text {cell }}=0\right)$. In these calculations, the polymer density $\sigma=2 / \mathrm{nm}^{2}$, the fraction of ligand $X_{L}=0.4, \varepsilon \beta=-0.4$ and $T=310 \mathrm{~K}$.

Figure 8 shows the variation of the ligand complex chain elongation at several $p H$ and $p K_{a}$ values. At a high $p H$, increasing the $p K_{a}$ values doesn't affect the chains length significantly. However, at local $p H$ of 5 , those chains with higher $p K_{a}$ values stretched significantly. In the figure, $\left\langle R_{L}\right\rangle$ reaches its maximum value at $p H=5$ and polyelectrolyte's $p K_{a}=8$. This figure demonstrates that the elongation of the polymer is very different near normal cells (with $p H \simeq 7$ ) than cancerous environment (with $p H \simeq 5$ ). Thus, to improve chain elongation and accordingly ligand-receptor binding, while screening healthy tissues, one can control these three parameters; $C_{\text {salt }}, p H$, and the $p K_{a}$ of the ligand complexes. The nanoparticle designer should look into the $C_{\text {salt }}$ and the $p H$ values at the site of administration, then design polymers that respond effectively (stretch) at the site. In a few words, designing ligand complexes with an effective $p K_{a}$ value depends significantly on the $C_{\text {salt }}$ and $p H$ of the site. Another technique could control the $C_{\text {salt }}$ at the site while delivering the nanoparticles to improve the targeted delivery.

\section{Cell surface charge}

The effect of cell charge on the length of ligand complexes is tested between the nanoparticle's surface and the cell surface, local $p H=6.5$, and minimal screening effect at $C_{\text {salt }}=0.4 \mathrm{M}$. The charges on the cell surface was varied between -0.1 and +0.1 with, $\sigma=0.3, X_{L}=0.4$ and $\varepsilon \beta=-0.3$. Figure 9 shows different elongation behaviors at different local ligand complex $p K_{a}$ values. There is almost no charge effect on $\langle R\rangle$ of weak polybases at $p K_{a}=3$. At $p K_{a}=5$, there is a slight increase in the ligand complexes length at a cell surface charge of $-0.1 \mathrm{e} / \mathrm{nm}^{2}$. Stronger polybases (ligand complexes) with $p K_{a}=7$ show a considerable increase on $\langle R\rangle$ at high negatively charged cell surface, and decreases steadily at cell charge above $+0.02 e / \mathrm{nm}^{2}$. The length of the ligand complexes increases dramatically at $p K_{a}=9$.

Using the previous parameters, the average volume fractions of all molecules in the system were studied to understand the chain elongation behavior that is due to the electrostatic effect. By investigating the volume fraction values at these different parameters for all molecules, they appear to be essentially unchanged except for

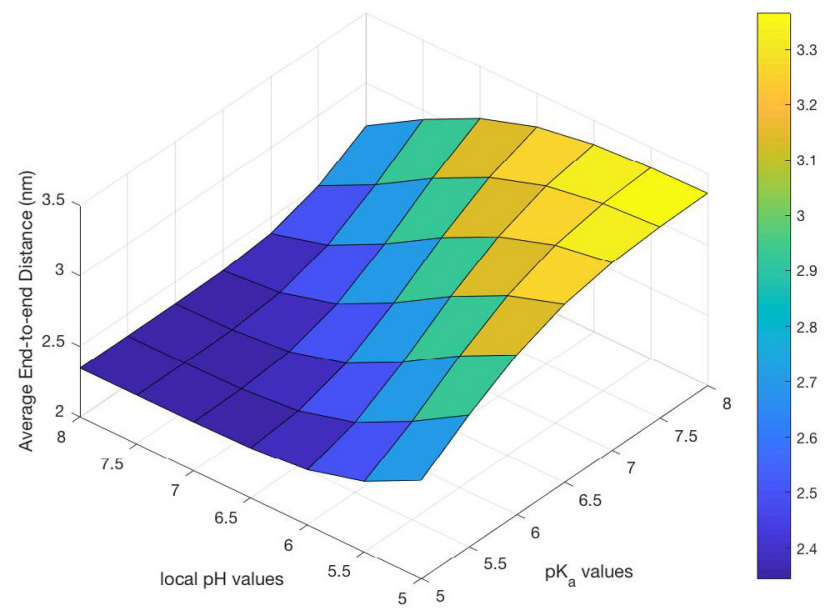

Figure 8: The effect of local $p H$ and cationic polyelectrolyte $p K_{a}$ on ligand complexes length. The surface plot indicate that decreasing the $\mathrm{pH}$ values causes the ligand complexes to stretch, while increasing the $p K_{a}$ values shows similar effect. The plot illustrates a maximum elongation at $\mathrm{pH}=5$ and $p K_{a}=8$ with $C_{\text {salt }}=0.4 \mathrm{M}$.

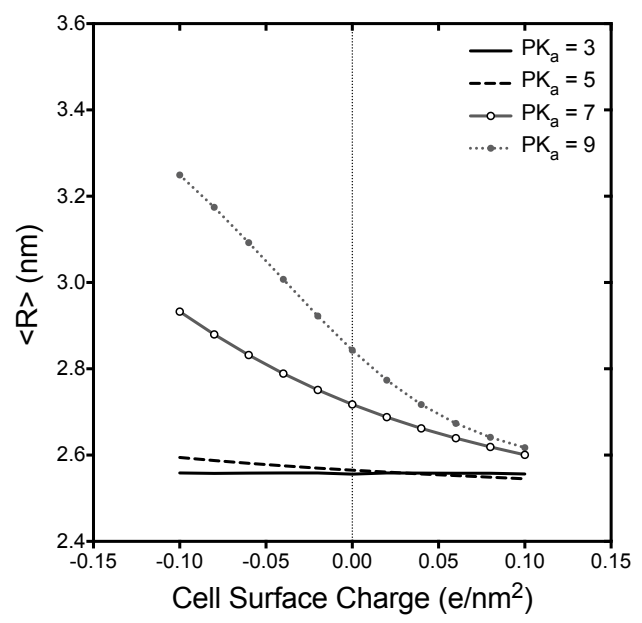

Figure 9: The effect of both the cellular charge density and $p K_{a}$ values on the ligand complexes length. Increasing the $p K_{a}$ values increases the length of the ligand complexes. This increase become significant near the negatively charged cell surface. 
the $\left\langle\phi_{L}(z)\right\rangle,\left\langle\phi_{L_{+}}(z)\right\rangle$, and $\left\langle\phi_{c l}(z)\right\rangle$ values. Figure 10 presents the $\left\langle\phi_{L_{+}}(z)\right\rangle$ at several $p K_{a}$ values and cell surface charge of $-0.1 e / \mathrm{nm}^{2}$. At $p K_{a}=5$, ligand complexes are almost uncharged. $\left\langle\phi_{L_{+}}(z)\right\rangle$ distribution does not vary along the different layers. At $p K_{a}=7$, more than a third of the ligand complex segments are charged. $\left\langle\phi_{L_{+}}(z)\right\rangle$ is uniformly distributed and at the last two layers near the cell surface, one can see a slight increase on its value near the

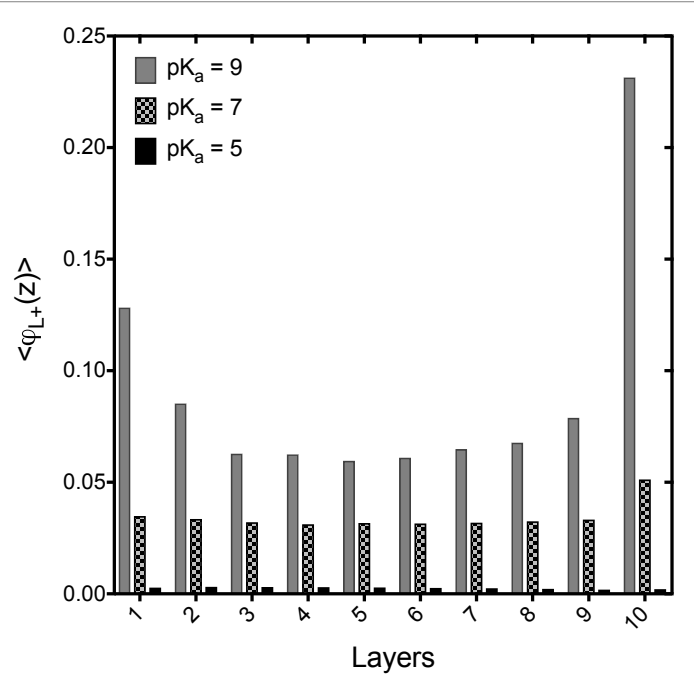

Figure 10: The average volume fraction of charged ligand complexes at negatively charge cell surface and several $p K_{a}$ values. At $p K_{a}=5$ the amount of $\left\langle\varphi_{L_{+}}\right\rangle$is insignificant, while it increases at $p K_{a}=7$ with uniform distribution among all layers and higher accumulation at the tenth layer. At $p K_{a}=9,\left\langle\varphi_{L+}\right\rangle$ values are more than doubled and the distribution varied among all layers. The accumulation is major at the tenth layer, which increases the binding probability.

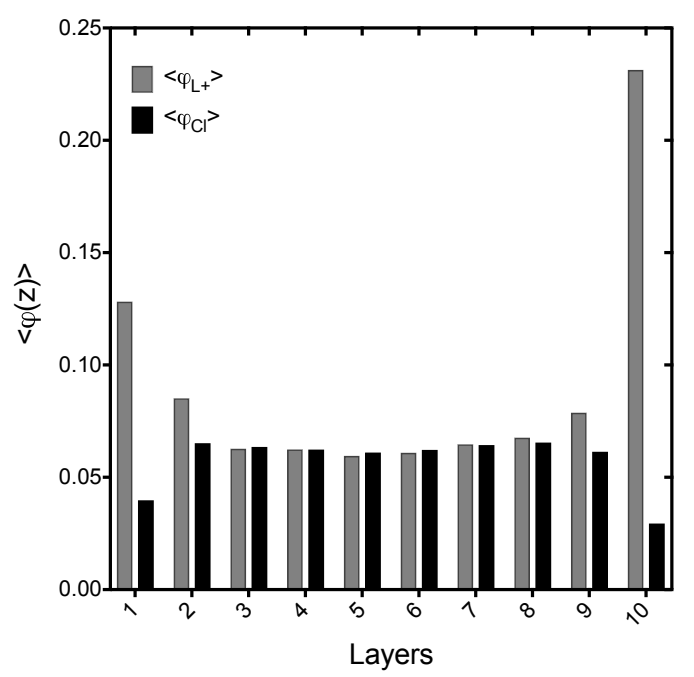

Figure 11: A comparison between the average volume fraction of charged ligand complexes and chloride ions at $p K_{a}=9$ and negatively charge cell surface. Both variables have the same values at the middle layers as chloride ions stabilizes the charged polymers. However, these two values vary near the surfaces due to the effect of the high density of monomers near the particle's surface and the negative charge on the cell surface. cell surface. $p K_{a}=9$, shows almost fully charged segments and a great accumulation near the negatively charged cell surface.

Figure 11 illustrates the average volume fraction of chloride ions $\left(\left\langle\phi_{c l}(z)\right\rangle\right)$ and $\left\langle\phi_{L_{+}}(z)\right\rangle$ at $p K_{a}=9 .\left\langle\phi_{c l}(z)\right\rangle$ decreases near the nanoparticles surface, which can be explained by the high density of molecules near the surface. In the middle layers, the $\left\langle\phi_{L_{+}}(z)\right\rangle$ and $\left\langle\phi_{c l}(z)\right\rangle$ are almost the same to balance the charges in the system. These two variables show an opposite behavior near the charged cell surface. However, there is a large accumulation of charge ligand complexes due to the negative charge of the cell surface.

\section{Density of ligands}

The total amount of monomers in the system is a conserved variable that is given by the following relation:

$$
\left(\sum_{z} \phi_{p}(z)=\sigma^{*} N^{*} v_{W}\right)
$$

where, $\sigma$ is in $\mathrm{nm}^{-2}$ units, and $v_{W}$ is the volume of water molecule, which is assumed to be the same for each molecule in the system, in units. The effect of the fraction of ligand complexes $\left(X_{L}\right)$ on the amount of bound ligands is studied at several polymers densities $\left(\sigma \simeq\{1,2,3\} \mathrm{nm}^{-2}\right)$. The fraction of ligand complexes $X_{L}$ is varied between (0.01 and 1.0). As the fraction of the surface covered by polybases increases, the fraction covered by shell-formers decreases. Other environmental parameters are set to mimic cancer cells (local $p H=5.5, C_{\text {salt }}=0.4 M, T=39^{\circ} \mathrm{C}$, and cell surface charge $\left.=-0.06 \mathrm{e} / \mathrm{nm}^{2}\right)$. Other design parameters are set at $p K_{a}=7$ and $\varepsilon \beta=-0.3$.

Figure 12 shows that the increase in ligands increases the number of bound ligands exponentially. A nanoparticle contains ligand complexes only at total surface density up to $\sigma=2 / \mathrm{nm}^{2}$ allows 0.24 fraction of bound ligands. Thus, at low surface density one can increase the ligands on the nanoparticle to increase the binding. Moreover, the figure shows that increasing the total polymers density increases the binding. However, at $\sigma=3 / \mathrm{nm}^{2}$ which is considered a very dense

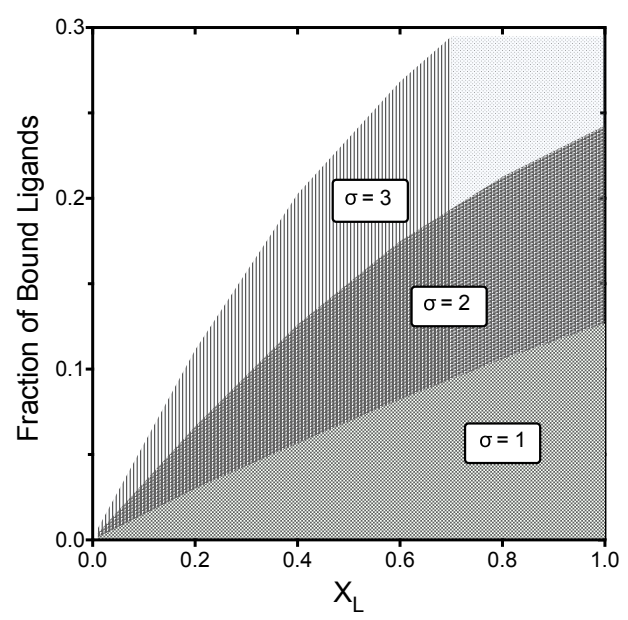

Figure 12: The effect of the nanoparticle ligand's density on the fraction of bound ligands on the cell surface at different polymers densities. 
surface and $X_{L}$ above 0.7 , no solution of the non-linear equations could be obtained. Note that the solution can be obtained by reducing the amount of charged molecules in the system, either by reducing $X_{L}$ or $C_{\text {salt }}$ vlaues. Due to the electrostatic forces, one can't increase the density of ligands that are highly charged at the chosen biological conditions. Thus, the presence of the shell-formers in the system seems to be very important to alleviate it. At high polymer density, the design shows that the existence of the shell-forming polymers is not only important for the system biocompatible properties, but also to improve the binding and stabilize the charged ligands on the nanoparticle.

\section{Conclusion}

The design of nanoparticles with ligand complexes that extend at a cancerous environment and collapse near healthy cells is a desired outcome of nanomedicine. The monoligand-receptor binding theory presented here supports the understanding of ligand complex elongation by analyzing the molecular interactions in a dense nonbulk system. It considers van der Waals, steric, and electrostatic interactions between all molecules at each layer of a system consisting of nine different species; water molecules $(\mathrm{W}), \mathrm{H}^{+}, \mathrm{OH}^{-}$salt ions $\left(\mathrm{Na}^{+}, \mathrm{Cl}^{-}\right)$, monomers of the shell-forming polymers $(S)$, uncharged and charged monomers that form the ligand complexes $\left(L, L_{+}\right)$, and bound ligandreceptors $(L R)$. The coupling between the three different interactions optimizes different structural organization of shell-forming polymers and ligand complexes. A decoupled mean-field approach is introduced in the theory to increase the accuracy of calculating van der Waals intermolecular interaction, and, as a result, the molecular reorganization. The molecular reorganization is influenced by different stimuli, such as temperature, $p H$, salt concentration, and charge density, as well as different nanoparticle's design parameters, such as polymers density, fraction of ligand complexes, choice of polybases $p K_{a}$, and elementary molecular interactions $\left(\varepsilon_{\text {intra }}, \varepsilon_{\text {inter }}\right)$. In this work, several stimuli and design parameters are studied explicitly to determine their effect on ligand complex elongation, which is vital for binding efficiency.

To design nanoparticles that target cancer cells only while screening healthy cells, one need to develop ligands that stretch at cancerous environment. Using polybases to design ligand complexes seems to be sufficient to improve the stretching and consequently the binding. However, the theory shows that the behavior of ligand complexes with different $p K_{a}$ deviate at different $p H$ and $C_{\text {salt }}$, as these three parameters control the chemical equilibrium and electrical forces in the system. In fact, one would think that increasing the salt concentration should screen the electrostatic interactions between ligand complex segments. However, in a dense system enclosed between two charged surfaces, this behavior is affected by the $p H$ and $p K_{a}$ values. At low $p K_{a}$ the salt ions can bind to the charged planes rather than the charged segments. Therefore, determining the biological conditions at the site is vital to design a nanoparticle with effective ligand complexes. The ligand complex should be designed specifically for specific site. This implies that one nanoparticle cannot work for different cancer cells.

Another design parameter is the total density or surface coverage of polymers $(\sigma)$. The theory shows that at low $(\sigma)$ one can design a nanoparticle with ligand complexes only-no shell-formers-to improve the binding. Moreover, increasing the surface coverage of all polymers on the nanoparticle seems to increase the binding as well. However, at high surface coverage, one can't design a nanoparticle with ligand complexes only due to the electrostatic forces between their charged segments themselves and the surrounding charged molecules. Thus, in the case of nanoparticle with high surface coverage, having shell-formers distributed on the nanoparticle surface beside the ligand complexes is significant to regulate the electrostatic forces in the system and increase the binding. Notice that in some nanoparticles, shell-formers are required to increase the solubility and improve the biocompatibility of the therapeutic nanoparticle. For such case, one should consider increasing the over all density and the fraction of ligands without affecting the stability of the system.

Future work should consider merging this design protocol that is provided for monoligand-nanoparticle with machine learning techniques to develop nanomedicine technology. Also, the study of the design of a nanoparticle with dual-ligand receptor should improve our understanding of the binding regulations.

\section{Acknowledgement}

The author is very grateful to Dr. M. Schick for a critical reading of the manuscript.

\section{References}

1. Grandin HM, Textor M (2012) Small Cargo. In Intelligent Biotechnology: Scientific and Engineering Concept, Enabling Technology, and Translation to Bio-Oriented Applications. John Wiley \& Sons, Inc, Hoboken, NJ, USA, pp: 255-256.

2. Galaev IY, Mattiasson B (1999) Smart polymers and what they could do in biotech- nology and medicine. Trends in Biotechnology 17: 335-340.

3. Chen G, Ito Y, Imanishi Y (1997) Regulation of growth and adhesion of cultured cells by insulin conjugated with thermoresponsive polymers. Biotechnol Bioeng 53: 339-344.

4. Lee ES, Gao Z, Kim D, Park K, Kwon IC, et al. (2008) Super pH-sensitive mul- tifunctional polymeric micelle for tumor pHe specific TAT exposure and multidrug resistance. J Controlled Release 129: 228-236.

5. Oishi M, Hayashi H, lijima M, Nagasaki $Y$ (2007) Endosomal release and intracellular delivery of anticancer drugs using $\mathrm{pH}$-sensitive PEGylated nanogels. J Materials Chem 17: 3720-3726.

6. Nasongkla N, Bey E, Ren J, Ai H, Khemtong C et al. (2006) Multifunctional Polymeric Micelles as Cancer-Targeted, MRI-Ultrasensitive Drug Delivery Systems. Nano Letters 6: 2427-2430.

7. Dhar S, Gu FX, Langer R, Farokhzad OC, Lippard SJ (2008) Targeted delivery of cisplatin to prostate cancer cells by aptamer functionalized Pt (IV) prodrugPLGA-PEG nanoparticles. Proc Natl Acad Sci USA 105: 17356-17361.

8. Ahn J, Miura Y, Yamada N, Chida Y, Liu X, et al. (2015) Antibody fragmentconjugated polymeric micelles incorporating platinum drugs for targeted therapy of pancreatic cancer. Biomaterials 39: $23-30$.

9. Wilhelm S, Tavares AJ, Dai Q, Ohta S, Audet J, et al. (2016) Analysis of nanoparticle delivery to tumours. Nature Reviews Materials 1: 16014-16113.

10. Martinelli E, De Palma R, Orditura M, De Vita F, Ciardiello F (2009) Antiepidermal growth factor receptor monoclonal antibodies in cancer therapy. Clin Exp Immunol 158: 1-9.

11. Hagy MC, Wang S, Dormidontova EE (2008) Optimization of Functionalized Polymer Layers for Specific Targeting of Mobile Receptors on Cell Surfaces. Langmuir 24: 13037-13047.

12. Longo G, Szleifer I (2005) Ligand-Receptor Interactions in Tethered Polymer Layers. Langmuir 21: 11342-11351.

13. Nap RJ, Szleifer I (2013) How to optimize binding of coated nanoparticles: coupling of physical interactions, molecular organization and chemical state. Biomater Sci 1: 814-911. 
Citation: Aldaais EA (2018) Generalized Theory of Monoligand-Receptor Binding for the Improvement of Nanoparticle Design. J Nanomed Nanotechnol 9: 512. doi: $10.4172 / 2157-7439.1000512$

Page 10 of 10

14. Abercrombie M, Ambrose E (1962) the surface properties of cancer cells: a review. Cancer Research 22: 525-548.

15. Chandra P, Noh HB, Shim YB (2013) Cancer cell detection based on the interac- tion between an anticancer drug and cell membrane components. Chemical Communications 49: 1900-1904.

16. Elsabahy M, Wooley KL (2012) Design of polymeric nanoparticles for biomedical delivery applications. Chem Soc Rev 41: 2545-2619.

17. Dobrzyn'ska I, Skrzydlewska E, Figaszewski ZA (2012) Changes in Electric Properties of Human Breast Cancer Cells. The J Membrane Biology 246: 161-166.

18. Aldaais EA (2016) A Theoretical Study of Polymer Based Drug Delivery Systems. University of South Carolina.
19. Rosenbluth MN, Rosenbluth AW (1955) Monte Carlo Calculation of the Average Extension of Molecular Chains. J Ch Ph 23: 356-365.

20. Voets IK, Leermakers FAM (2008) Self-consistent field theory for obligatory coassem- bly. Physical Review E 78: 061801-15.

21. Tagliazucchi M, de la Cruz MO, Szleifer I (2010) Self-organization of grafted polyelec- trolyte layers via the coupling of chemical equilibrium and physical interactions. Proceedings of the National Academy of Sciences 107: 5300-5305.

22. Szleifer I, Carignano MA (1996) Tethered Polymer Layers. In Advances in Chemical Physics. John Wiley \& Sons, Inc, Hoboken, NJ, USA, PP: 165-260. 